Research Paper

\title{
The Association of ERCCI and ERCC5 Polymorphisms with Lung Cancer Risk in Han Chinese
}

\author{
Xueling $\mathrm{Lan}^{1}$, Ying $\mathrm{Li}^{2}$, Yefeng $\mathrm{Wu}^{3}$, Xia $\mathrm{Li}^{2}$, Lan $\mathrm{Xu}^{1}$ \\ 1. Department of Laboratory, Cancer Hospital of China Medical University, Liaoning Cancer Hospital \& Institute, Shenyang, Liaoning 110042, China. \\ 2. Department of Radiation Oncology, Cancer Hospital of China Medical University, Liaoning Cancer Hospital \& Institute, and Key Laboratory of Tumor \\ Radiosensitization and Normal Tissue Radioprotection of Liaoning Province, Shenyang, Liaoning 110042, China. \\ 3. Central Laboratory, Cancer Hospital of China Medical University, Liaoning Cancer Hospital \& Institute, Shenyang, Liaoning 110042, China. \\ $\triangle$ Corresponding author: Lan Xu, E-mail: xulan@CanceHosp-LN-CMU.com.
}

(C) The author(s). This is an open access article distributed under the terms of the Creative Commons Attribution License (https://creativecommons.org/licenses/by/4.0/). See http://ivyspring.com/terms for full terms and conditions.

Received: 2021.02.10; Accepted: 2021.11.30; Published: 2022.01.01

\begin{abstract}
Background: Polymorphisms in DNA damage repair genes are important determinants for cancer susceptibility, clinical phenotype diversity, and therapy. However, their relationship with lung cancer remains unclear. This study aimed to investigate the role of DNA damage repair gene polymorphisms in the risk of lung cancer.

Methods: The matrix-assisted laser desorption ionization-time of flight (MALDI-TOF) mass spectroscopybased genotyping system was used to genotype 601 individuals (200 lung cancer patients and 401 age- and sex-matched healthy controls) for polymorphisms in excision repair cross-complementing group 1 ( $E R C C l)$ and ERCC5 genes.

Results: The ERCC5 rs4771436 GG genotype, recessive model (GG vs. GT+TT), and the ERCC5 rs 1047768 recessive model ( $C C$ vs. $C T+T T)$ were associated with significantly increased risks of lung cancer $(P=0.029$, $P=0.014$, and $P=0.044$, respectively), especially in men and individuals aged 60 years or younger.

Conclusion: ERCC5 rs4771436 and rs1047768 genotypes were associated with an increased risk of lung cancer, suggesting that polymorphisms in DNA repair genes are significantly related to the risk of lung cancer, and play an important role in the occurrence of lung cancer.
\end{abstract}

Key words: ERCC; polymorphisms; lung cancer; risk

\section{Introduction}

Lung cancer is one of the common malignant tumors in the world, and it remains the leading cause of cancer mortality because of its high malignant and metastatic potential [1]. Epidemiological studies of migrant populations point to a role for environmental and/or lifestyle factors in cancer etiology [2-6]. The occurrence of lung cancer is closely related to smoking, as shown by its observed downward trend in global incidence with the launch of anti-smoking campaigns; however, it still ranks first among all cancer types. In recent years, in addition to environmental factors, genetic factors have become a hot spot in the etiology of lung cancer.

Alterations in the DNA damage repair pathway are hallmarks of cancer [7], and the relationships between such pathways and cancer are varied and complex. DNA repair pathways are essential for preventing DNA damage from causing mutations and cytotoxicity [8], but the incorrect repair of DNA lesions often leads to carcinogenesis and genomic instability [7]. An important connection linking the DNA damage repair pathway to cancer development is variations in DNA damage repair genes.

Single nucleotide polymorphisms (SNPs) are the most common type of genetic variation, and participate in carcinogenesis. SNPs in genes encoding proteins involved in DNA damage repair pathways are associated with the risk and prognosis of various cancers, including lung cancer. For example, the X-ray repair cross-complementing protein 1 gene (XRCC1) codon 399 Gln allele and TP53 codon 72 Arg allele appear to have a protective effect against lung adenocarcinoma, especially in individuals older than 50 years of age [9]. Moreover, excision repair 
cross-complementing group 1 gene (ERCC1) rs3212986 GG homozygosity and rs11615 T allele were associated with a higher risk of developing non-small cell lung cancer (NSCLC) in the Polish population [10]. ERCC2 rs13181 and ERCC1 rs3212986 SNPs have an elevated association with lung cancer risk $[2,11]$, while the O6-methylguanine-DNA methyltransferase gene SNP rs12917 is associated with an increased risk of lung cancer [12]. Additionally, the ERCC2 rs13181 TG genotype and rs1799793 CT genotype significantly increased the risk of cancer death [13]. The identification of these SNPs could be a useful low-cost tool for evaluating individual cancer risk, promoting the earlier detection and management of cancer.

A complex DNA repair machinery has evolved to protect genomic integrity in the face of a myriad of DNA damage sources. If DNA repair fails, this damage can lead to carcinogenesis and tumor genomic instability [14]. Genetic and epigenetic aberrations in DNA damage repair pathway genes are associated with various pathogeneses [15-22]. These changes may be useful biomarkers in a liquid biopsy for the early detection and prevention of lung cancer. Here, we investigated the link between SNPs in DNA damage repair pathway genes and susceptibility to lung cancer by studying three ERCC1 and two ERCC5 SNPs in a Chinese Han population.

\section{Materials and Methods}

\section{Study design and study population}

This study design was approved by the Human Ethics Committee of Liaoning Cancer Hospital (Shenyang, China). Each participant provided their written informed consent during an epidemiological investigation. A total of 200 lung cancer patients were recruited from Liaoning Cancer Hospital who had undergone surgical resection or needle biopsy diagnosis/treatment between 2018 and 2019. A total of 401 age- and sex-matched healthy controls were recruited from a health check program in Liaoning Province between 2018 and 2019. All diagnoses were based on histopathological examinations. Information about smoking status, alcohol consumption, and family history were acquired in a face-to-face questionnaire survey. Fasting venous blood was obtained from participants and stored at $-20^{\circ} \mathrm{C}$.

To evaluate the relationship between SNPs and clinicopathological parameters of lung cancer, histology or clinical data were assessed according to World Health Organization criteria, and tumor-nodemetastasis (TNM) staging was performed according to the $8^{\text {th }}$ edition of the International Union against Cancer/American Joint Committee on Cancer 2017 criteria [23].

\section{SNP selection}

A compilation of the genes involved in the DNA damage repair pathway was conducted on the basis of a published panel of DNA damage repair genes [24-27] and NCBI-Gene website analysis (https:// www.ncbi.nlm.nih.gov/gene/). We selected the following five SNPs for analysis in this study: ERCC1 rs735482, rs11615, and rs3212986 and ERCC5 rs4771436 and rs1047768.

\section{SNP genotyping}

Genomic DNA was extracted from peripheral blood samples obtained from study participants using the phenol/chloroform method according to our standard procedure [28]. Matrix-assisted laser desorption ionization-time of fight (MALDI-TOF) mass spectroscopy-based genotyping was used to genotype all 601 individuals for SNPs in the five DNA damage repair genes.

\section{Statistical analysis}

Statistical analysis was performed using SPSS statistical software (version 22.0). Adjusted odds ratios and 95\% confidence intervals (CIs) for the relationship between SNPs and lung cancer risk were calculated by multivariable logistic regression, with adjustment for sex and age. In the analysis stratified by sex, the age was adjusted and vice versa. The $\chi^{2}$ test was used to evaluate the relationship between polymorphism genotypes and the clinicopathological parameters of lung cancer patients. Logistic regression was used for the interaction and epistatic effect analysis of ERCC1 and ERCC5 polymorphisms in the risk of lung cancer. Haplotype-base risk prediction of SNPs in ERCC1 and ERCC5 genes for lung cancer was performed using the HaploView (https://www.broadinstitute.org/scientificcommunity/science/programs/medical-and-populat ion-genetics/haploview/haploview).

\section{Results}

\section{Baseline patient characteristics}

A comparison of baseline characteristics is shown in Table 1. There was a significant difference in age distribution between lung cancer patients and controls, but not with respect to sex. The mean age and mean age of menarche also differed significantly between patients and controls (both $P<0.001$ ). The mean menopausal age in patients was 60.50 years and only a small proportion had a family history of cancer (14.1\%). Regarding tumor invasion depth, $45.8 \%$ and $54.2 \%$ of patients were in T1-2 and T3-4, respectively. Tumor stages I-II (10.1\%) and III-IV (89.9\%) accounted 
for most lung cancer cases, and $80.5 \%$ of patients had positive lymph nodes while $63.6 \%$ had metastasis.

Table 1. The baseline characteristics of the objects

\begin{tabular}{|c|c|c|c|}
\hline Characteristics & Cases & Controls & $P$ value \\
\hline Sample size & 200 & 401 & \\
\hline Age & & & $<0.001$ \\
\hline Mean \pm SD & $58.76 \pm 9.60$ & $36.25 \pm 12.63$ & \\
\hline Mmenarche & 60.5 & 32 & \\
\hline Range & $27-80$ & $17-73$ & \\
\hline \multicolumn{4}{|l|}{ Gender } \\
\hline Female & $75(37.5 \%)$ & $175(43.6 \%)$ & 0.150 \\
\hline Male & $125(62.5 \%)$ & $226(56.4 \%)$ & \\
\hline \multicolumn{4}{|l|}{ T stage } \\
\hline $1-2$ & $60(45.8 \%)$ & & \\
\hline $3-4$ & $71(54.2 \%)$ & & \\
\hline \multicolumn{4}{|l|}{ N stage } \\
\hline Negative & $29(19.5 \%)$ & & \\
\hline Positive & $120(80.5 \%)$ & & \\
\hline \multicolumn{4}{|l|}{ M stage } \\
\hline Negative & $71(36.4 \%)$ & & \\
\hline Positive & $124(63.6 \%)$ & & \\
\hline \multicolumn{4}{|l|}{ Clinical stage } \\
\hline I-II & $20(10.1 \%)$ & & \\
\hline III-IV & $178(89.9 \%)$ & & \\
\hline \multicolumn{4}{|l|}{ Smoking } \\
\hline No & $117(58.5 \%)$ & & \\
\hline Yes & $83(41.5 \%)$ & & \\
\hline \multicolumn{4}{|l|}{ Drinking } \\
\hline No & $42(21.0 \%)$ & & \\
\hline Yes & $158(79.0 \%)$ & & \\
\hline \multicolumn{4}{|c|}{ Family history of cancer } \\
\hline No & $170(85.9 \%)$ & & \\
\hline Yes & $28(14.1 \%)$ & & \\
\hline \multicolumn{4}{|l|}{ Pathological type } \\
\hline Small cell cancer & $57(30.0 \%)$ & & \\
\hline Squamous carcinoma & $37(19.5 \%)$ & & \\
\hline Adenocarcinoma & $96(50.5 \%)$ & & \\
\hline \multicolumn{4}{|l|}{ Ki67 } \\
\hline$\leq 50$ & $5(26.3 \%)$ & & \\
\hline$>50$ & $14(73.7 \%)$ & & \\
\hline \multicolumn{4}{|l|}{ EGFR } \\
\hline Wild type & $19(35.8 \%)$ & & \\
\hline Mutation type & $34(64.2 \%)$ & & \\
\hline \multicolumn{4}{|l|}{ SCC } \\
\hline Normal & $57(78.1 \%)$ & & \\
\hline Increased & $16(21.9 \%)$ & & \\
\hline \multicolumn{4}{|l|}{ CEA } \\
\hline Normal & 95 (50.3\%) & & \\
\hline Increased & $94(49.7 \%)$ & & \\
\hline \multicolumn{4}{|l|}{ CYFRA } \\
\hline Normal & 68 (43.6\%) & & \\
\hline Increased & $88(56.4 \%)$ & & \\
\hline \multicolumn{4}{|l|}{ NSE } \\
\hline Normal & $35(23.2 \%)$ & & \\
\hline Increased & $116(76.8 \%)$ & & \\
\hline \multicolumn{4}{|l|}{ PRO } \\
\hline Normal & $22(45.8 \%)$ & & \\
\hline Increased & $26(54.2 \%)$ & & \\
\hline \multicolumn{4}{|l|}{ TAP } \\
\hline Normal & $4(8.7 \%)$ & & \\
\hline Increased & $42(91.3 \%)$ & & \\
\hline TK1 & & & \\
\hline Normal & $5(71.4 \%)$ & & \\
\hline Increased & $2(28.6 \%)$ & & \\
\hline
\end{tabular}

Association of ERCCI and ERCC5 SNPs with lung cancer risk

Multivariable logistic regression was used to investigate the association of ERCC1 and ERCC5 SNPs with lung cancer risk. ERCC5 rs4771436 and rs1047768 had a significant association with lung cancer risk progression (Table 2). Specifically, we found that carriers of the ERCC5 rs4771436 GG genotype, the recessive model (GG vs. GT+TT) and the ERCC5 rs1047768 CC genotype, the recessive model (CC vs. $\mathrm{CT}+\mathrm{TT}$ ) showed a significantly increased risk of lung cancer $(P<0.05)$. However, there was no significant association between ERCC1 SNPs and lung cancer risk progression.

Table 2. The association of ERCCI and ERCC5 polymorphisms with lung cancer risk

\begin{tabular}{|c|c|c|c|c|c|c|}
\hline Genetype & SNP & Cases & Controls & $P$ value & $P$ value & OR $(95 \% \mathrm{CI})$ \\
\hline \multirow{6}{*}{ ERCC1 } & $\begin{array}{l}\text { rs735482 } \\
\end{array}$ & $\mathrm{N}=199$ & $\mathrm{~N}=400$ & 0.367 & & \\
\hline & AA & $61(30.5 \%)$ & $124(31.0 \%)$ & & / & 1(Ref) \\
\hline & CA & $107(53.5 \%)$ & $196(49.0 \%)$ & & 0.161 & $1.49(0.85,2.58)$ \\
\hline & $\mathrm{CC}$ & $31(15.5 \%)$ & $80(20.0 \%)$ & & 0.537 & $0.79(0.38,1.66)$ \\
\hline & $\begin{array}{l}\mathrm{CA}+\mathrm{CC} \text { vs. } \\
\mathrm{AA}\end{array}$ & / & I & & 0.375 & $1.27(0.75,2.14)$ \\
\hline & $\begin{array}{l}\text { CC vs. } \\
C A+A A\end{array}$ & I & I & & 0.153 & $0.62(0.32,1.19)$ \\
\hline \multirow[t]{6}{*}{ ERCC1 } & rs11615 & $\mathrm{N}=200$ & $\mathrm{~N}=400$ & 0.299 & & \\
\hline & AA & $18(9.0 \%)$ & $24(6.0 \%)$ & & / & 1(Ref) \\
\hline & GA & $67(33.5 \%)$ & $151(37.8 \%)$ & & 0.620 & $0.77(0.28,2.16)$ \\
\hline & GG & $115(57.5 \%)$ & $225(56.3 \%)$ & & 0.946 & $0.97(0.36,2.60)$ \\
\hline & $\begin{array}{l}\text { GA+GG vs. } \\
\text { AA }\end{array}$ & / & / & & 0.799 & $0.88(0.34,2.32)$ \\
\hline & $\begin{array}{l}\text { GG vs. } \\
\text { GA+AA }\end{array}$ & / & I & & 0.507 & $1.18(0.72,1.92)$ \\
\hline \multirow[t]{6}{*}{ ERCC1 } & rs3212986 & $\mathrm{N}=199$ & $\mathrm{~N}=396$ & 0.809 & & \\
\hline & $\mathrm{CC}$ & $95(47.7 \%)$ & $187(47.2 \%)$ & & / & 1(Ref) \\
\hline & CA & $83(41.7 \%)$ & $173(43.7 \%)$ & & 0.993 & $1.00(0.60,1.66)$ \\
\hline & $\mathrm{AA}$ & $21(10.6 \%)$ & $36(9.1 \%)$ & & 0.812 & $1.11(0.48,2.55)$ \\
\hline & $\begin{array}{l}\text { CA+AA vs. } \\
\text { CC }\end{array}$ & / & 1 & & 0.942 & $1.02(0.63,1.65)$ \\
\hline & $\begin{array}{l}\text { AA vs. } \\
\text { CA+CC }\end{array}$ & / & / & & 0.799 & $1.11(0.51,2.45)$ \\
\hline \multirow[t]{6}{*}{ ERCC5 } & rs4771436 & $\mathrm{N}=198$ & $\mathrm{~N}=396$ & 0.616 & & \\
\hline & TT & $104(52.5 \%)$ & $207(52.3 \%)$ & & / & 1(Ref) \\
\hline & GT & $78(39.4 \%)$ & $165(41.7 \%)$ & & 0.498 & $0.84(0.50,1.40)$ \\
\hline & GG & $16(8.1 \%)$ & $24(6.1 \%)$ & & 0.029 & $2.89(1.11,7.53)$ \\
\hline & $\begin{array}{l}\text { GT+GG vs. } \\
\text { TT }\end{array}$ & 1 & / & & 0.951 & $1.02(0.63,1.64)$ \\
\hline & $\begin{array}{l}\text { GG vs. } \\
\text { GT+TT }\end{array}$ & / & / & & 0.014 & $3.25(1.26,8.36)$ \\
\hline \multirow[t]{6}{*}{ ERCC5 } & rs1047768 & $\mathrm{N}=200$ & $\mathrm{~N}=396$ & 0.391 & & \\
\hline & TT & $105(52.5 \%)$ & $197(49.7 \%)$ & & / & \\
\hline & CT & $72(36.0 \%)$ & $163(41.2 \%)$ & & 0.181 & $0.70(0.41,1.18)$ \\
\hline & $\mathrm{CC}$ & $23(11.5 \%)$ & $36(9.1 \%)$ & & 0.105 & $2.09(0.86,5.08)$ \\
\hline & $\mathrm{CT}+\mathrm{CC}$ vs. TT & / & / & & 0.550 & $0.86(0.53,1.40)$ \\
\hline & CC vs. $\mathrm{CT}+\mathrm{TT}$ & / & / & & 0.044 & $2.40(1.02,5.61)$ \\
\hline
\end{tabular}

\section{Stratified analysis of ERCCI and ERCC5 SNPs with lung cancer risk}

Using stratified analysis, we showed that the ERCC5 rs4771436 GG genotype, the recessive model (GG vs. GT+TT) and ERCC5 rs1047768 CC genotype, the recessive model (CC vs. CT+TT) conferred 5.01-fold, 5.39-fold, 3.06-fold, and 3.25-fold increases in lung cancer progression, respectively, in patients aged $\leq 60$ years. In older individuals (aged $>60$ years), no genotype was significantly correlated with the risk of lung cancer. In men, the ERCC5 rs1047768 the 
recessive model (CC vs. CT+TT) conferred a 3.00-fold increase in lung cancer progression. However, no SNPs were significantly associated with the risk of lung cancer in women. These results are shown in Table 3.

Table 3. Stratified analysis of the association of ERCCl and ERCC5 polymorphisms with lung cancer risk

\begin{tabular}{|c|c|c|c|c|c|c|c|c|c|c|c|c|c|}
\hline Genetype & SNP & Cases & Controls & $P$ value & $P$ value & OR $(95 \% \mathrm{CI})$ & & $\mathrm{TT}$ & $59(12.3 \%)$ & $188(39.2 \%)$ & & / & 1 (Ref) \\
\hline Age $>60$ & & & & & & & & $\mathrm{CT}$ & $28(5.8 \%)$ & $157(32.8 \%)$ & & 0.073 & $0.57(0.31,1.05)$ \\
\hline \multirow[t]{8}{*}{ ERCC1 } & rs735482 & $\mathrm{N}=100$ & $\mathrm{~N}=17$ & 0.499 & & & & $\mathrm{CC}$ & $13(2.7 \%)$ & $34(7.1 \%)$ & & 0.034 & $3.06(1.09,8.63)$ \\
\hline & $\mathrm{AA}$ & $30(25.6 \%)$ & $6(5.1 \%)$ & & / & $1(\operatorname{Ref})$ & & $\mathrm{CT}+\mathrm{CC}$ & / & / & & 0.383 & $0.78(0.46,1.35)$ \\
\hline & $\mathrm{CA}$ & $50(42.7 \%)$ & $6(5.1 \%)$ & & 0.348 & $1.82(0.52,6.39)$ & & vs. TT & & & & & \\
\hline & $\mathrm{CC}$ & $20(17.1 \%)$ & $5(4.3 \%)$ & & 0.809 & $0.85(0.22,3.22)$ & & CC vs. & / & / & & 0.012 & $3.25(1.29,8.19)$ \\
\hline & $\mathrm{CA}+\mathrm{CC}$ & / & / & & 0.558 & $1.39(0.46,4.20)$ & & $\mathrm{CT}+\mathrm{TT}$ & & & & & \\
\hline & vs. AA & & & & & & Male & & & & & & \\
\hline & CC vs. & / & / & & 0.378 & $0.59(0.18,1.90)$ & ERCC1 & rs735482 & $\mathrm{N}=125$ & $N=225$ & 0.104 & & \\
\hline & $\mathrm{CA}+\mathrm{AA}$ & & & & & & & $\mathrm{AA}$ & $39(11.1 \%)$ & $76(21.7 \%)$ & & / & 1(Ref) \\
\hline \multirow[t]{7}{*}{ ERCC1 } & rs11615 & $\mathrm{N}=100$ & $\mathrm{~N}=17$ & 0.360 & & & & CA & $67(19.1 \%)$ & $97(27.7 \%)$ & & 0.264 & $1.53(0.73,3.22)$ \\
\hline & $\mathrm{AA}$ & $11(9.4 \%)$ & $1(0.9 \%)$ & & / & 1(Ref) & & $\mathrm{CC}$ & $19(5.4 \%)$ & $52(14.9 \%)$ & & 0.393 & $0.66(0.25,1.71)$ \\
\hline & GA & $37(31.6 \%)$ & $4(3.4 \%)$ & & 0.808 & $1.36(0.11,16.18)$ & & $\mathrm{CA}+\mathrm{CC}$ & / & / & & 0.590 & $1.21(0.61,2.40)$ \\
\hline & GG & $52(44.4 \%)$ & $12(10.3 \%)$ & & 0.424 & $0.41(0.05,3.62)$ & & vs. AA & & & & & \\
\hline & $\begin{array}{l}\text { GA+GG } \\
\text { vs. AA }\end{array}$ & / & / & & 0.610 & $0.57(0.07,4.88)$ & & $\begin{array}{l}C C \text { vs. } \\
C A+A A\end{array}$ & / & / & & 0.131 & $0.52(0.22,1.22)$ \\
\hline & GG vs. & / & / & & 0.139 & $0.43(0.14,1.32)$ & ERCC1 & rs11615 & $\mathrm{N}=125$ & $\mathrm{~N}=225$ & 0.405 & & \\
\hline & $\mathrm{GA}+\mathrm{AA}$ & & & & & & & $\mathrm{AA}$ & $14(4.0 \%)$ & $16(4.6 \%)$ & & / & $1($ Ref $)$ \\
\hline \multirow[t]{7}{*}{ ERCC1 } & rs3212986 & $\mathrm{N}=100$ & $\mathrm{~N}=17$ & 0.821 & & & & GA & $40(11.4 \%)$ & $79(22.6 \%)$ & & 0.500 & $0.65(0.19,2.27)$ \\
\hline & $\mathrm{CC}$ & $55(47.0 \%)$ & $8(6.8 \%)$ & & / & 1 (Ref) & & GG & $71(20.3 \%)$ & $130(37.1 \%)$ & & 0.798 & $0.86(0.27,2.77)$ \\
\hline & $\mathrm{CA}$ & $36(30.8 \%)$ & $7(6.0 \%)$ & & 0.538 & $0.70(0.23,2.16)$ & & GA+GG & / & / & & 0.665 & $0.78(0.24,2.46)$ \\
\hline & $\mathrm{AA}$ & $9(7.7 \%)$ & $2(1.7 \%)$ & & 0.553 & $0.59(0.10,3.37)$ & & vs. AA & & & & & \\
\hline & $\begin{array}{l}\mathrm{CA}+\mathrm{AA} \\
\text { vs. CC }\end{array}$ & / & / & & 0.481 & $0.69(0.24,1.95)$ & & $\begin{array}{l}\text { GG vs. } \\
\text { GA+AA }\end{array}$ & / & / & & 0.547 & $1.22(0.64,2.35)$ \\
\hline & AA vs. & / & / & & 0.676 & $0.70(0.13,3.71)$ & ERCC1 & rs3212986 & $\mathrm{N}=125$ & $\mathrm{~N}=222$ & 0.981 & & \\
\hline & $\mathrm{CA}+\mathrm{CC}$ & & & & & & & $\mathrm{CC}$ & $59(17.0 \%)$ & $105(30.0 \%)$ & & / & 1 (Ref) \\
\hline \multirow[t]{7}{*}{ ERCC5 } & rs4771436 & $\mathrm{N}=98$ & $\mathrm{~N}=17$ & 0.388 & & & & $\mathrm{CA}$ & $56(16.1 \%)$ & $98(28.2 \%)$ & & 0.965 & $1.02(0.52,1.98)$ \\
\hline & $\mathrm{TT}$ & $51(44.3 \%)$ & $9(7.8 \%)$ & & / & 1(Ref) & & AA & $10(2.9 \%)$ & $19(5.5 \%)$ & & 0.780 & $1.19(0.35,3.98)$ \\
\hline & GT & $43(37.4 \%)$ & $6(5.2 \%)$ & & 0.503 & $1.49(0.46,4.82)$ & & $\mathrm{CA}+\mathrm{AA}$ & / & / & & 0.906 & $1.04(0.55,1.99)$ \\
\hline & GG & $4(3.5 \%)$ & $2(1.7 \%)$ & & 0.263 & $0.32(0.05,2.34)$ & & vs. CC & & & & & \\
\hline & $\begin{array}{l}\text { GT+GG } \\
\text { vs. TT }\end{array}$ & / & / & & 0.820 & $1.13(0.39,3.30)$ & & $\begin{array}{l}\text { AA vs. } \\
C A+C C\end{array}$ & / & / & & 0.778 & $1.19(0.36,3.88)$ \\
\hline & GG vs. & 0.1 & / & & 0.189 & $0.29(0.04,1.86)$ & ERCC5 & rs4771436 & $\mathrm{N}=124$ & $\mathrm{~N}=222$ & 0.077 & & \\
\hline & $\mathrm{GT}+\mathrm{TT}$ & & & & & & & $\mathrm{TT}$ & $75(21.7 \%)$ & $113(32.7 \%)$ & & / & 1(Ref) \\
\hline \multirow[t]{7}{*}{ ERCC5 } & rs1047768 & $\mathrm{N}=100$ & $\mathrm{~N}=17$ & 0.798 & & & & GT & $38(11.0 \%)$ & $95(27.5 \%)$ & & 0.070 & $0.51(0.25,1.06)$ \\
\hline & $\mathrm{TT}$ & $46(39.3 \%)$ & $9(7.7 \%)$ & & / & $1(\operatorname{Ref})$ & & GG & $11(3.2 \%)$ & $14(4.0 \%)$ & & 0.186 & $2.39(0.66,8.73)$ \\
\hline & $\mathrm{CT}$ & $44(37.6 \%)$ & $6(5.1 \%)$ & & 0.540 & $1.42(0.46,4.41)$ & & $\mathrm{GT}+\mathrm{GG}$ & / & / & & 0.234 & $0.67(0.35,1.29)$ \\
\hline & $\mathrm{CC}$ & $10(8.5 \%)$ & $2(1.7 \%)$ & & 0.960 & $1.05(0.18,5.93)$ & & vs. TT & & & & & \\
\hline & $\begin{array}{l}\mathrm{CT}+\mathrm{CC} \\
\text { vs. TT }\end{array}$ & / & I & & 0.642 & $1.28(0.45,3.63)$ & & $\begin{array}{l}\text { GG vs. } \\
\text { GT+TT }\end{array}$ & / & / & & 0.063 & $3.52(0.94,13.22)$ \\
\hline & CC vs. & / & / & & 0.746 & $0.76(0.14,4.11)$ & ERCC5 & rs1047768 & $\mathrm{N}=125$ & $\mathrm{~N}=223$ & 0.420 & & \\
\hline & $\mathrm{CT}+\mathrm{TT}$ & & & & & & & $\mathrm{TT}$ & $63(18.1 \%)$ & $112(32.2 \%)$ & & / & 1(Ref) \\
\hline Age $\leq 60$ & & & & & & & & $\mathrm{CT}$ & $43(12.4 \%)$ & $87(25.0 \%)$ & & 0.359 & $0.71(0.34,1.48)$ \\
\hline \multirow[t]{8}{*}{ ERCC1 } & rs735482 & $\mathrm{N}=99$ & $\mathrm{~N}=383$ & 0.126 & & & & $\mathrm{CC}$ & $19(5.5 \%)$ & $24(6.9 \%)$ & & 0.095 & $2.54(0.85,7.59)$ \\
\hline & AA & $31(6.4 \%)$ & $118(24.5 \%)$ & & / & 1 (Ref) & & $\mathrm{CT}+\mathrm{CC}$ & / & / & & 0.905 & $0.96(0.50,1.84)$ \\
\hline & $\mathrm{CA}$ & $57(11.8 \%)$ & $190(39.4 \%)$ & & 0.279 & $1.40(0.76,2.60)$ & & vs. TT & & & & & \\
\hline & $\mathrm{CC}$ & $11(2.3 \%)$ & $75(15.6 \%)$ & & 0.763 & $0.87(0.35,2.16)$ & & CC vs. & / & / & & 0.042 & $3.00(1.04,8.68)$ \\
\hline & $\mathrm{CA}+\mathrm{CC}$ & / & / & & 0.450 & $1.26(0.69,2.28)$ & & $\mathrm{CT}+\mathrm{TT}$ & & & & & \\
\hline & vs. AA & & & & & & Female & & & & & & \\
\hline & CC vs. & / & / & & 0.296 & $0.66(0.30,1.44)$ & ERCC1 & rs735482 & $\mathrm{N}=74$ & $\mathrm{~N}=175$ & 0.924 & & \\
\hline & $\mathrm{CA}+\mathrm{AA}$ & & & & & & & AA & $22(8.8 \%)$ & $48(19.3 \%)$ & & / & 1 (Ref) \\
\hline \multirow[t]{7}{*}{ ERCC1 } & rs11615 & $\mathrm{N}=100$ & $N=383$ & 0.301 & & & & CA & $40(16.1 \%)$ & $99(39.8 \%)$ & & 0.410 & $1.42(0.62,3.23)$ \\
\hline & AA & $7(1.4 \%)$ & $23(4.8 \%)$ & & / & 1 (Ref) & & $\mathrm{CC}$ & $12(4.8 \%)$ & $28(11.2 \%)$ & & 0.938 & $1.05(0.32,3.46)$ \\
\hline & GA & $30(6.2 \%)$ & $147(30.4 \%)$ & & 0.540 & $0.70(0.23,2.16)$ & & $\mathrm{CA}+\mathrm{CC}$ & / & / & & 0.460 & $1.36(0.60,3.06)$ \\
\hline & GG & $63(13.0 \%)$ & $213(44.1 \%)$ & & 0.665 & $1.31(0.39,4.45)$ & & vs. AA & & & & & \\
\hline & $\begin{array}{l}\text { GA+GG } \\
\text { vs. AA }\end{array}$ & / & / & & 0.977 & $0.98(0.32,3.04)$ & & $\begin{array}{l}\text { CC vs. } \\
C A+A A\end{array}$ & / & / & & 0.665 & $0.80(0.30,2.18)$ \\
\hline & GG vs. & / & / & & 0.112 & $1.58(0.90,2.77)$ & ERCC1 & rs11615 & $\mathrm{N}=75$ & $\mathrm{~N}=175$ & 0.743 & & \\
\hline & $\mathrm{GA}+\mathrm{AA}$ & & & & & & & AA & $4(1.6 \%)$ & $8(3.2 \%)$ & & / & $1(\operatorname{Ref})$ \\
\hline \multirow[t]{5}{*}{ ERCC1 } & rs3212986 & $\mathrm{N}=99$ & $\mathrm{~N}=379$ & 0.397 & & & & GA & $27(10.8 \%)$ & $72(28.8 \%)$ & & 0.897 & $1.13(0.17,7.56)$ \\
\hline & $\mathrm{CC}$ & $40(8.4 \%)$ & $179(37.4 \%)$ & & / & 1(Ref) & & GG & $44(17.6 \%)$ & $95(38.0 \%)$ & & 0.798 & $1.28(0.19,8.44)$ \\
\hline & $\mathrm{CA}$ & $47(9.8 \%)$ & $166(34.7 \%)$ & & 0.764 & $1.09(0.62,1.93)$ & & $\mathrm{GA}+\mathrm{GG}$ & / & / & & 0.838 & $1.21(0.19,7.55)$ \\
\hline & $\mathrm{AA}$ & $12(2.5 \%)$ & $34(7.1 \%)$ & & 0.574 & $1.31(0.52,3.30)$ & & vs. AA & & & & & \\
\hline & $\begin{array}{l}\mathrm{CA}+\mathrm{AA} \\
\text { vs. CC }\end{array}$ & / & / & & 0.683 & $1.12(0.65,1.94)$ & & $\begin{array}{l}\text { GG vs. } \\
\text { GA+AA }\end{array}$ & / & / & & 0.748 & $1.13(0.54,2.34)$ \\
\hline
\end{tabular}




\begin{tabular}{|c|c|c|c|c|c|c|}
\hline Genetype & SNP & Cases & Controls & $P$ value & $P$ value & OR $(95 \% \mathrm{CI})$ \\
\hline \multirow{6}{*}{ ERCC1 } & rs3212986 & $\mathrm{N}=74$ & $\mathrm{~N}=174$ & 0.412 & & \\
\hline & CC & $36(14.5 \%)$ & $82(33.1 \%)$ & & / & 1 (Ref) \\
\hline & CA & $27(10.9 \%)$ & $75(30.2 \%)$ & & 0.976 & $0.99(0.45,2.16)$ \\
\hline & AA & $11(4.4 \%)$ & $17(6.9 \%)$ & & 0.963 & $1.03(0.32,3.30)$ \\
\hline & $\begin{array}{l}\mathrm{CA}+\mathrm{AA} \\
\text { vs. CC }\end{array}$ & / & / & & 0.986 & $0.99(0.48,2.05)$ \\
\hline & $\begin{array}{l}\text { AA vs. } \\
\text { CA+CC }\end{array}$ & / & / & & 0.935 & $1.05(0.36,3.06)$ \\
\hline \multirow[t]{6}{*}{ ERCC5 } & rs4771436 & $\mathrm{N}=74$ & $\mathrm{~N}=174$ & 0.099 & & \\
\hline & TT & $29(11.7 \%)$ & $94(37.9 \%)$ & & / & 1(Ref) \\
\hline & GT & $40(16.1 \%)$ & $70(28.2 \%)$ & & 0.323 & $1.47(0.69,3.14)$ \\
\hline & GG & $5(2.0 \%)$ & $10(4.0 \%)$ & & 0.073 & $3.65(0.89,14.99)$ \\
\hline & $\begin{array}{l}\text { GT+GG } \\
\text { vs. TT }\end{array}$ & / & / & & 0.164 & $1.67(0.81,3.43)$ \\
\hline & $\begin{array}{l}\text { GG vs. } \\
\text { GT+TT }\end{array}$ & l & / & & 0.108 & $3.00(0.78,11.46)$ \\
\hline \multirow[t]{6}{*}{ ERCC5 } & rs1047768 & $\mathrm{N}=75$ & $\mathrm{~N}=173$ & 0.597 & & \\
\hline & TT & $42(16.9 \%)$ & $85(34.3 \%)$ & & / & 1 (Ref) \\
\hline & CT & $29(11.7 \%)$ & $76(30.6 \%)$ & & 0.347 & $0.70(0.33,1.48)$ \\
\hline & CC & $4(1.6 \%)$ & $12(4.8 \%)$ & & 0.654 & $1.43(0.30,6.77)$ \\
\hline & $\begin{array}{l}\mathrm{CT}+\mathrm{CC} \\
\text { vs. TT }\end{array}$ & / & / & & 0.447 & $0.76(0.37,1.55)$ \\
\hline & $\begin{array}{l}\text { CC vs. } \\
\text { CT+TT }\end{array}$ & l & / & & 0.561 & $1.56(0.35,6.89)$ \\
\hline
\end{tabular}

\section{Association of ERCCI and ERCC5 SNPs with clinicopathological parameters of lung cancer patients}

Among the SNPs associated with an increased risk of lung cancer, ERCC1 rs735482 in the recessive model was significantly related to pathological type.
Moreover, the heterozygous genotype of ERCC1 rs11615 and ERCC5 rs1047768 in the recessive model were significantly related to sex, while the heterozygous genotype and ERCC5 rs4771436 in the dominant model and ERCC5 rs1047768 in the recessive model were significantly related to smoking. Other SNPs had no significant correlation with clinicopathological parameters. All results are shown in Table 4.

\section{The interaction and epistatic effect analysis and HaploView in the risk of lung cancer}

In the logistic regression analysis, the interaction and epistatic effects were not found, and all results are shown in Table 5 and Table 6. Haplotype-base risk prediction of SNPs in ERCC1 and ERCC5 genes for lung cancer was performed using the HaploView. ERCC1 rs4771436 and rs1047768 were highly linked, and ERCC5 rs735482 and rs11615, rs3212986 and rs11615 were also highly linked, forming haplotype blocks ( $\mathrm{D}^{\prime}>0.95$ ). Haplotype block were T-C, G-T, T-T, C-A, A-A, C-G, A-G, C-A, A-G, C-G, respectively. There were no significant statistical differences in this analysis. All results were presented in Table 7.

Table 4. The association of ERCCl and ERCC5 polymorphisms with clinicopathological parameters of lung cancer patients

\begin{tabular}{|c|c|c|c|c|c|c|c|c|c|c|c|c|c|c|c|c|c|c|c|c|c|c|c|c|c|c|c|c|c|c|c|c|c|c|c|}
\hline & & $\mathrm{C} 1 \mathrm{r}$ & 87354 & & & & & & $\mathrm{CC} 1 \mathrm{rs}$ & 11615 & & & & & ERC & $\mathrm{Cl}_{1 \mathrm{rs}}$ & 32129 & & & & & ERC & $\mathrm{C} 5 \mathrm{rs}$ & 47714 & & & & & ERC & $\mathrm{C} 5 \mathrm{rs}$ & : 10477 & & & & \\
\hline 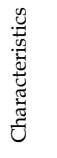 & $\frac{\pi}{3}$ & 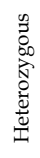 & 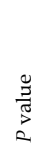 & 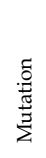 & $\begin{array}{l}\text { בे } \\
\text { है } \\
\text { ह } \\
\text { E. }\end{array}$ & 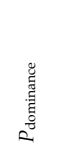 & 旁 & $\frac{\pi}{3}$ & 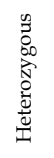 & $\begin{array}{l}\frac{\pi}{\pi} \\
\frac{5}{\pi} \\
2 \\
2\end{array}$ & 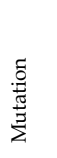 & 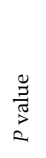 & 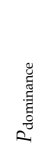 & 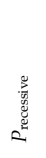 & $\frac{\pi}{3}$ & 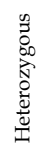 & 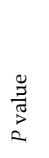 & 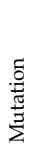 & 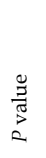 & 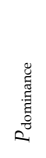 & 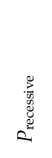 & $\frac{\pi}{3}$ & 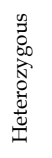 & 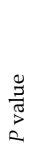 & 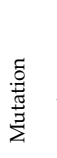 & 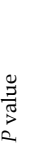 & 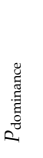 & 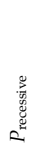 & $\frac{\pi}{3}$ & 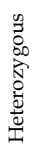 & 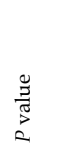 & 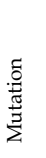 & 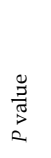 & 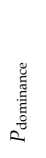 & 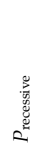 \\
\hline 茄 & & & $\begin{array}{l}\stackrel{\circ}{\stackrel{0}{0}} \\
\stackrel{0}{0}\end{array}$ & & $\begin{array}{l}\text { त్ } \\
\text { గ్ర }\end{array}$ & 各 & స్ & & & ํㅗㅇ & & $\begin{array}{l}m_{0} \\
\stackrel{0}{0}\end{array}$ & $\begin{array}{l}\mathbb{J} \\
0 \\
0\end{array}$ & గ్ర్త్ర & & & : & & है & ने & Iี & & & 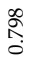 & & 离 & 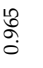 & $\begin{array}{l}\text { Pे } \\
\text { t. }\end{array}$ & & & 莺 & & $\begin{array}{l}\vec{D} \\
\stackrel{2}{0}\end{array}$ & 命 & 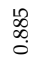 \\
\hline 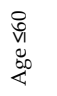 & शे & A & & $\infty$ & & & & ल & 토 & & ล゚ & & & & సे & $\stackrel{m}{\vec{n}}$ & & 난 & & & & I্] & 壱 & & मे & & & & ন্ & 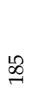 & & भि & & & \\
\hline 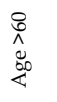 & $\infty$ & เ̊ & & מิ & & & & $\approx$ & $F$ & & तु & & & & 8 & $\mathscr{F}$ & & $F$ & & & & 8 & के & & 0 & & & & 㔛 & in & & $\approx$ & & & \\
\hline 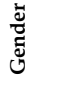 & & & 足 & & $\begin{array}{l}\stackrel{\wp}{\aleph} \\
\text { م. }\end{array}$ & 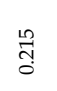 & $\underset{ }{\stackrel{9}{\circ}}$ & & & क्ष & & $\begin{array}{l}\stackrel{J}{0} \\
\stackrel{\sigma}{0}\end{array}$ & $\begin{array}{l}\text { t' } \\
\stackrel{0}{0}\end{array}$ & 圆 & & & 啇 & & $\begin{array}{l}\overrightarrow{m_{0}} \\
\stackrel{0}{0}\end{array}$ & ồ. & 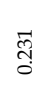 & & & 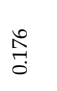 & & 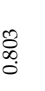 & 范 & 令 & & & 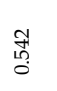 & & $\stackrel{\widetilde{\tilde{O}}}{\circ}$ & $\begin{array}{l}\text { त゙ } \\
\text { o } \\
0\end{array}$ & $\stackrel{\hat{0}}{0}$ \\
\hline 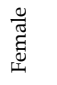 & 尺 & ले & & q & & & & $\approx$ & s & & ले & & & & $\stackrel{\infty}{=}$ & 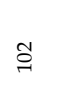 & & $\stackrel{\sim}{\sim}$ & & & & $\widetilde{\Xi}$ & $\stackrel{9}{\exists}$ & & $\stackrel{2}{2}$ & & & & $\widehat{\widehat{ح}}$ & 总 & & $\stackrel{2}{\sim}$ & & & \\
\hline$\frac{\stackrel{0}{\varpi}}{\Sigma}$ & $\stackrel{\mathscr{Z}}{\Rightarrow}$ & 总 & & $\Sigma$ & & & & के & $\exists$ & & సี & & & & $\underset{\sim}{\stackrel{d}{\sigma}}$ & 苛 & & নి & & & & $\stackrel{\infty}{\stackrel{\infty}{\sim}}$ & $\stackrel{\mathscr{m}}{\stackrel{2}{2}}$ & & $\ddot{2}$ & & & & 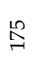 & $\stackrel{ి}{7}$ & & 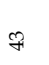 & & & \\
\hline 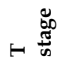 & & & ત્ત & & $\begin{array}{l}\text { वे } \\
\stackrel{f}{0}\end{array}$ & 苞 & 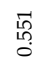 & & & ஜ̊ & & 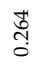 & 悉 & 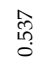 & & & $\stackrel{8}{\stackrel{0}{0}}$ & & 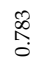 & $\begin{array}{l}\text { तે } \\
\text { ণิ }\end{array}$ & F & & & $\begin{array}{l}\text { 佫 } \\
\text { on }\end{array}$ & & 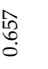 & $\begin{array}{l}\text { 胥 } \\
\text { 。 }\end{array}$ & 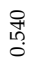 & & & 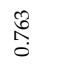 & & 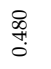 & ڤ̊. & శ్ \\
\hline & 2 & 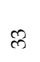 & & $\Lambda$ & & & & $a$ & $\exists$ & & ले & & & & เุ & ते & & in & & & & 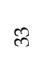 & สి & & $\Lambda$ & & & & ल & $\bar{\lambda}$ & & in & & & \\
\hline
\end{tabular}




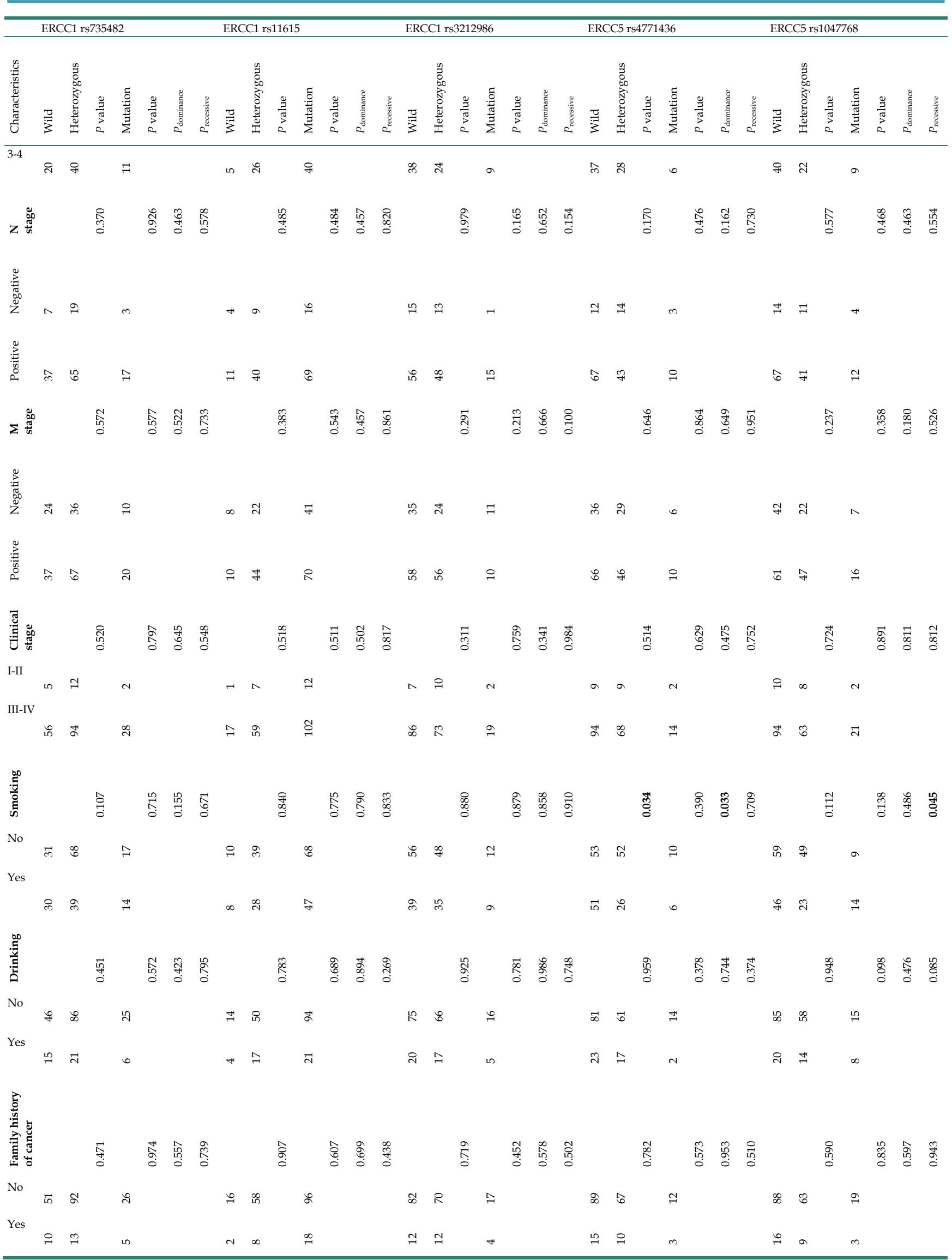




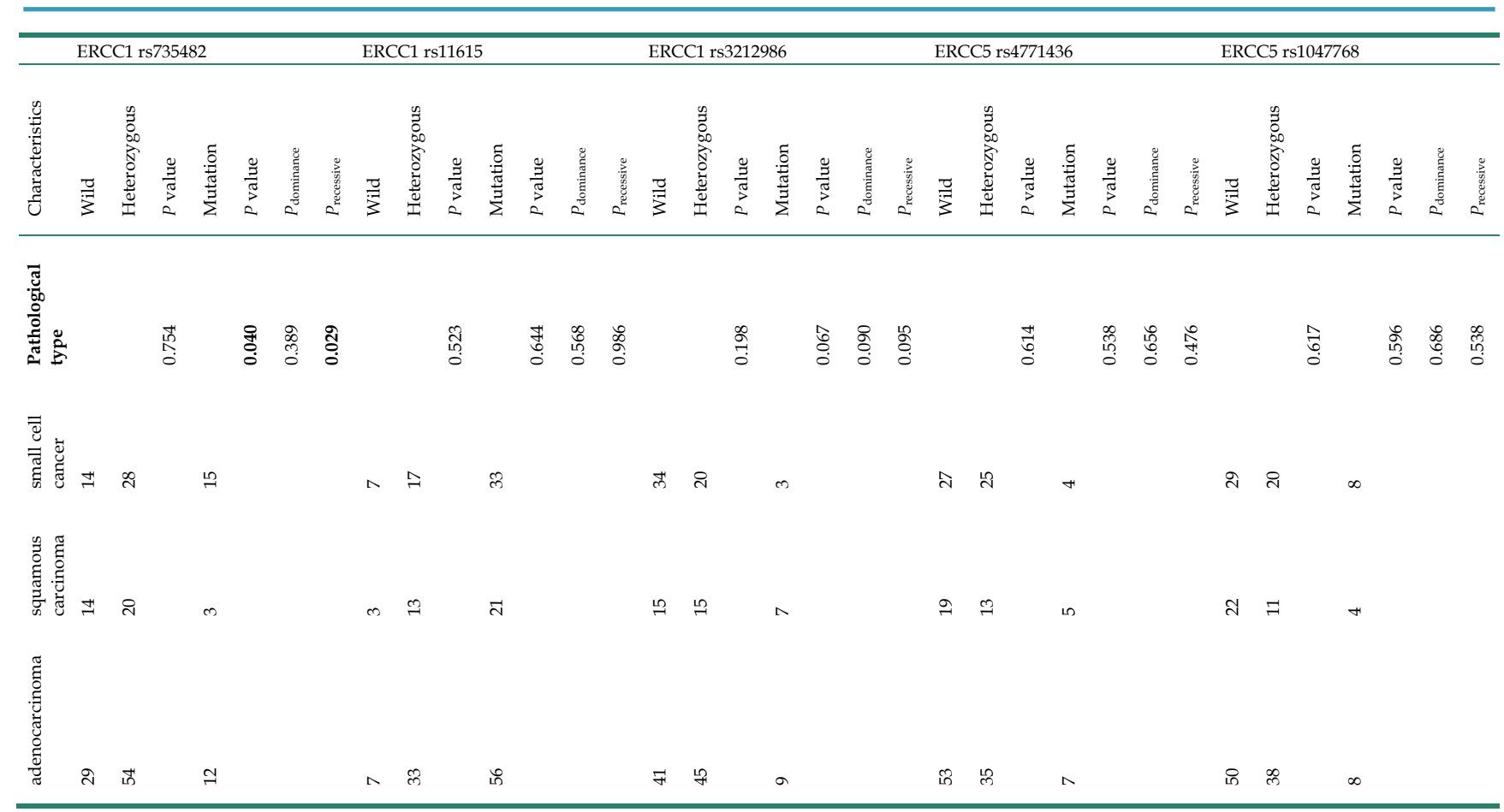

Table 5. The interaction of ERCCI and ERCC5 polymorphisms in the risk of lung cancer

\begin{tabular}{|c|c|c|c|c|c|c|c|c|c|c|c|}
\hline & & \multicolumn{2}{|c|}{ ERCC1 rs735482 } & \multicolumn{2}{|l|}{ ERCC1 rs11615 } & \multicolumn{2}{|c|}{ ERCC1 rs3212986 } & \multicolumn{2}{|c|}{ ERCC5 rs4771436 } & \multicolumn{2}{|c|}{$\begin{array}{l}\text { ERCC5 } \\
\text { rs1047768 }\end{array}$} \\
\hline & & $\overline{\mathrm{CC}}$ & $\mathrm{CA}+\mathrm{AA}$ & GG & $\mathrm{GA}+\mathrm{AA}$ & AA & $\mathrm{CA}+\mathrm{CC}$ & GG & GT+TT & $\mathrm{CC}$ & $\mathrm{CT}+\mathrm{TT}$ \\
\hline \multicolumn{12}{|c|}{ ERCC1 rs735482 } \\
\hline \multirow[t]{2}{*}{ CC } & Case/Control & / & / & / & / & / & / & / & / & / & / \\
\hline & OR $(95 \% \mathrm{CI})$ & / & I & / & I & / & l & / & / & / & / \\
\hline \multirow{3}{*}{$\begin{array}{l}\mathrm{CA}+ \\
\mathrm{AA}\end{array}$} & Case/Control & / & / & / & / & / & / & / & / & / & / \\
\hline & OR $(95 \% \mathrm{CI})$ & / & / & / & / & I & / & I & / & I & / \\
\hline & & / & & / & & / & & / & & / & \\
\hline \multicolumn{12}{|c|}{ ERCC1 rs11615 } \\
\hline \multirow[t]{2}{*}{ GG } & Case/Control & $27 / 71$ & $87 / 154$ & / & l & / & I & / & I & / & I \\
\hline & OR $(95 \% \mathrm{CI})$ & 1 (Ref) & $1.21(0.83,1.76)$ & l & l & l & l & l & l & l & / \\
\hline \multirow{3}{*}{$\begin{array}{l}\text { GA+ } \\
\text { AA }\end{array}$} & Case/Control & $4 / 9$ & $78 / 166$ & / & / & / & / & / & / & / & / \\
\hline & OR $(95 \% \mathrm{CI})$ & $\begin{array}{l}0.96 \\
(0.29,3.21)\end{array}$ & $0.69(0.18,2.56)$ & / & / & / & / & / & / & / & / \\
\hline & & $\mathrm{P}=0.574$ & & / & & / & & / & & / & \\
\hline \multicolumn{12}{|c|}{ ERCC1 rs3212986 } \\
\hline \multirow[t]{2}{*}{ AA } & Case/Control & $0 / 0$ & $21 / 36$ & $21 / 36$ & $0 / 0$ & / & / & / & I & / & / \\
\hline & OR $(95 \% \mathrm{CI})$ & 1 (Ref) & $1.16(0.65,2.07)$ & 1 (Ref) & $1.18(0.65,2.14)$ & l & / & l & / & l & / \\
\hline \multirow{3}{*}{$\begin{array}{l}\mathrm{CA}+ \\
\mathrm{CC}\end{array}$} & Case/Control & $31 / 78$ & $144 / 281$ & $94 / 185$ & $84 / 174$ & / & / & / & / & / & / \\
\hline & OR $(95 \% \mathrm{CI})$ & $\begin{array}{l}0.76 \\
(0.48,1.21)\end{array}$ & NA & $1.04(0.73,1.50)$ & NA & / & / & / & / & / & / \\
\hline & & 0.608 & & 0.583 & & / & & / & & / & \\
\hline \multicolumn{12}{|c|}{ ERCC5 rs4771436 } \\
\hline \multirow[t]{2}{*}{ GG } & Case/Control & $1 / 6$ & $15 / 18$ & $6 / 16$ & $10 / 8$ & $1 / 1$ & $15 / 23$ & / & I & / & I \\
\hline & OR $(95 \% \mathrm{CI})$ & 1 (Ref) & $1.64(0.80,3.36)$ & 1 (Ref) & $2.75(1.04,7.25)$ & 1 (Ref) & $1.33(0.68,2.63)$ & / & / & / & I \\
\hline \multirow{3}{*}{$\begin{array}{l}\text { GT+ } \\
\text { TT }\end{array}$} & Case/Control & $29 / 73$ & $149 / 299$ & $107 / 206$ & $75 / 166$ & $20 / 34$ & $161 / 333$ & / & / & / & I \\
\hline & OR $(95 \% \mathrm{CI})$ & $\begin{array}{l}0.78 \\
(0.48,1.25)\end{array}$ & $0.26(0.03,2.24)$ & $1.14(0.80,1.64)$ & $2.60(0.07,1.02)$ & $1.25(0.70,2.25)$ & $1.28(0.07,23.59)$ & / & / & / & I \\
\hline & & 0.247 & & 0.054 & & 0.868 & & / & & l & \\
\hline \multicolumn{12}{|c|}{ ERCC5 rs1047768 } \\
\hline \multirow[t]{2}{*}{ CC } & Case/Control & $5 / 7$ & $18 / 29$ & $12 / 23$ & $11 / 13$ & $3 / 5$ & $20 / 31$ & $0 / 0$ & $23 / 36$ & / & I \\
\hline & OR $(95 \% \mathrm{CI})$ & 1 (Ref) & $1.19(0.64,2.22)$ & 1 (Ref) & $1.78(0.76 .4 .16)$ & 1 (Ref) & $1.31(0.72,2.37)$ & 1 (Ref) & $\begin{array}{l}1.32 \\
(0.75,2.30)\end{array}$ & / & / \\
\hline \multirow{3}{*}{$\begin{array}{l}\text { CT+ } \\
\text { TT }\end{array}$} & Case/Control & $26 / 72$ & $147 / 288$ & $103 / 201$ & $74 / 159$ & $18 / 31$ & $158 / 324$ & $16 / 24$ & $159 / 332$ & / & I \\
\hline & OR $(95 \% \mathrm{CI})$ & $\begin{array}{l}0.69 \\
(0.42,1.13)\end{array}$ & $1.70(0.43,6.77)$ & $1.09(0.76,1.57)$ & $0.57(0.19,1.77)$ & $1.23(0.66,2.26)$ & $0.76(0.15,3.99)$ & $1.38(0.71,2.67)$ & NA & / & l \\
\hline & & 0.454 & & 0.332 & & 0.745 & & 0.345 & & / & \\
\hline
\end{tabular}


Table 6. Epistatic effect analysis of ERCCI and ERCC5 polymorphisms with lung cancer risk

\begin{tabular}{|c|c|c|c|c|}
\hline \multirow[t]{2}{*}{ SNP1 } & \multirow[t]{2}{*}{ SNP2 } & \multirow[t]{2}{*}{ SNP3 } & \multicolumn{2}{|c|}{$\mathrm{CON}$ vs $\mathrm{CA}$} \\
\hline & & & $P$ value & OR $(95 \% \mathrm{CI})$ \\
\hline rs735482 & rs11615 & rs3212986 & 0.897 & $1.04(0.56,1.92)$ \\
\hline rs735482 & rs11615 & rs4771436 & 0.323 & $1.39(0.72,2.69)$ \\
\hline rs735482 & rs11615 & rs1047768 & 0.307 & $1.34(0.77,2.33)$ \\
\hline rs735482 & rs3212986 & rs4771436 & 0.333 & $1.39(0.72,2.68)$ \\
\hline rs735482 & rs3212986 & rs1047768 & 0.337 & $1.31(0.75,2.29)$ \\
\hline rs11615 & rs3212986 & rs4771436 & 0.345 & $1.37(0.71,2.64)$ \\
\hline rs11615 & rs3212986 & rs1047768 & 0.382 & $1.28(0.74,2.23)$ \\
\hline rs3212986 & rs4771436 & rs1047768 & 0.339 & $1.31(0.75,2.29)$ \\
\hline
\end{tabular}

Table 7. Haplotype-base risk prediction of SNPs in ERCC1 and ERCC5 genes for lung cancer

\begin{tabular}{|c|c|c|c|c|c|c|c|}
\hline Gene & SNPs & Haplotype & Modela $^{a}$ & F value & $\mathrm{T}$ value & OR & P value \\
\hline \multirow[t]{6}{*}{ ERCC1 } & rs4771436-rs1047768 & $\mathrm{TC}$ & Unadjusted & 0.297 & 0.011 & 0.986 & 0.917 \\
\hline & & & Adjusted & 0.297 & 0.139 & 1.070 & 0.709 \\
\hline & rs4771436-rs1047768 & GT & Unadjusted & 0.271 & 0.103 & 1.050 & 0.748 \\
\hline & & & Adjusted & 0.271 & 0.056 & 0.954 & 0.813 \\
\hline & rs4771436-rs1047768 & $\mathrm{TT}$ & Unadjusted & 0.430 & 0.021 & 0.982 & 0.885 \\
\hline & & & Adjusted & 0.430 & 0.015 & 0.979 & 0.903 \\
\hline \multirow[t]{8}{*}{ ERCC5 } & rs735482-rs11615 & CA & Unadjusted & 0.021 & 0.027 & 1.090 & 0.869 \\
\hline & & & Adjusted & 0.021 & 0.024 & 1.120 & 0.877 \\
\hline & rs735482-rs11615 & AA & Unadjusted & 0.230 & 0.059 & 1.040 & 0.808 \\
\hline & & & Adjusted & 0.230 & 0.301 & 0.893 & 0.583 \\
\hline & rs735482-rs11615 & CG & Unadjusted & 0.417 & 0.546 & 0.909 & 0.460 \\
\hline & & & Adjusted & 0.417 & 0.056 & 0.957 & 0.813 \\
\hline & rs735482-rs11615 & AG & Unadjusted & 0.332 & 0.245 & 1.070 & 0.621 \\
\hline & & & Adjusted & 0.332 & 0.484 & 1.140 & 0.486 \\
\hline \multirow[t]{6}{*}{ ERCC5 } & rs3212986-rs11615 & CA & Unadjusted & 0.253 & 0.043 & 1.030 & 0.835 \\
\hline & & & Adjusted & 0.253 & 0.307 & 0.896 & 0.580 \\
\hline & rs3212986-rs11615 & AG & Unadjusted & 0.311 & 0.034 & 1.020 & 0.855 \\
\hline & & & Adjusted & 0.311 & 1.030 & 0.030 & 0.863 \\
\hline & rs3212986-rs11615 & CG & Unadjusted & 0.437 & 0.131 & 0.955 & 0.718 \\
\hline & & & Adjusted & 0.437 & 0.117 & 1.060 & 0.732 \\
\hline
\end{tabular}

\section{Discussion}

DNA damage repair pathways play an important role in the occurrence and development of cancer, especially in lung cancer which has high morbidity and mortality. Cancer cells carry various types of mutations and show the aberrant expression of genes involved in DNA repair responses, leading to genome instability, the promotion of carcinogenesis, and cancer progression. Defects in DNA repair responses have been considered suitable biomarkers for cancer risk screening [29]. The association of ERCC genetic variation with lung cancer has been widely evaluated worldwide [17, 30], but has been rarely reported in the Han Chinese population, especially in Liaoning Province.

ERCC polymorphisms are also known to be closely related to the occurrence and development of other cancers. For instance, ERCC3 rs4150434 and ERCC5 rs4771436 and rs2094258 SNPs were previously associated with genetic susceptibility to lung cancer [31], ERCC5 rs2296147 was associated with a reduced risk of esophageal cancer [32], and ERCC2 rs1799793 was positively associated with prostate cancer risk in an Asian population [16]. Moreover, five SNPs (rs1047768, rs2227869, rs1047768, rs17655, and rs2227869) of ERCC5, a gene involved in nucleotide excision repair, were associated with a reduced stomach cancer risk [33].

Of course, there are also genetic polymorphisms that affect the risk of lung cancer by affecting ERCC mutations, such as rs229614 and rs17655, which may be one of the molecular mechanisms of lung cancer [30]. Other polymorphisms are also significantly associated with the risk of lung cancer; for example those in XRCC1 and TP53, especially in individuals aged over 50 years, whose detection allows the earlier diagnosis of disease [9]. ERCC1 and XRCC1 polymorphisms have also been significantly associated with the risk of lung cancer, especially in non-smokers [2-5]. Additionally, Chaszczewska et al. reported that a nuclear factor kappa B subunit 2 polymorphism may be associated with NSCLC risk in the Polish population, and is a potential marker for NSCLC in men [10]. Moreover, a XRCC1 polymorphism was closely related to the incidence of NSCLC, especially in women [3]. In the high incidence region of Hebei Province, the C/C genotype of XPC exon 15 appears to increase the risk of developing esophageal squamous cell carcinoma in the non-smoking population [6]. Polymorphisms in DNA repair genes may be related to an increased risk of malignant transformation in lung cancer, especially among smokers and residents of coal mining areas 
[34].

Our findings suggest that ERCC5 might be a candidate gene for lung cancer susceptibility in the Han Chinese population. We report for the first time a significant association between ERCC5 SNPs rs4771436 and rs1047768 with lung cancer risk progression in Liaoning Province. We found that carriers of the ERCC5 rs4771436 GG genotype, the recessive model (GG vs. GT+TT) and the ERCC5 rs1047768 CC genotype, the recessive model (CC vs. $\mathrm{CT}+\mathrm{TT}$ ) had increased risks of lung cancer. Our findings provide experimental evidence to support the use of ERCC1 and ERCC5 SNPs as potential biomarkers of specific types of lung cancer.

We conducted stratified analyses in our study to examine how age and sex affected the correlation between SNPs and the risk of lung cancer. We found that the ERCC5 rs4771436 GG genotype, the recessive model (GG vs. GT+TT) and the ERCC5 rs1047768 CC genotype, the recessive model (CC vs. CT+TT) conferred increases in lung cancer progression in individuals aged $\leq 60$ years. Additionally, the ERCC5 rs1047768 the recessive model (CC vs. CT+TT) conferred an increase in lung cancer progression in men. These results are consistent with reported findings, although potential underlying mechanisms require further investigation.

Liu et al. previously detected a correlation between the tumor stage of lung cancer patients and ERCC1 SNP rs3212986 [5]. Furthermore, the tumor necrosis factor receptor superfamily, member 19 gene plays an inhibitory role in lung cancer, and its differential expression is significantly related to tumor TNM staging [35]. Clinicopathological parameters such as age, sex, smoking status, and tumor stage are associated with the distribution of genetic polymorphisms and the risk of tumor incidence. In the present study, we compared the genotype distribution of the five SNPs in lung cancer patients with different clinicopathological parameters. We found that ERCC1 rs735482 in the recessive model was significantly related to pathological type, being least common among patients with squamous cell carcinoma. Moreover, the heterozygous genotype of ERCC1 rs11615 and ERCC5 rs1047768 in the recessive model were significantly related to sex, with the heterozygous ERCC1 rs11615 genotype being most widely distributed among men and the mutation genotype of ERCC5 rs1047768 least common among women. Finally, the heterozygous genotype of ERCC5 rs4771436 and this SNP in the dominant model together with ERCC5 rs1047768 in the recessive model were significantly related to smoking. Other SNPs had no significant correlation with clinicopathological parameters. Because these results derived from a correlation study, they should be confirmed by conducting basic experiments.

In addition, we have further done SNPs-SNPs interaction, epistatis effect and haplotype analysis. ERCC1 and ERCC5 are located on chromosome 13 and chromosome 19, respectively. ERCC1 rs4771436 and rs1047768 were highly linked, and ERCC5 rs735482 and rs11615, rs3212986 and rs11615 were also highly linked, forming haplotype blocks ( $\mathrm{D}^{\prime}$ $>0.95$ ). Haplotype block were T-C, G-T, T-T, C-A, A-A, C-G, A-G, C-A, A-G, C-G, respectively. However, there were no significant statistical differences.

Some limitations should be considered in our study. First, the sample size was relatively small, especially of lung cancer patients, so our findings need further confirmation in larger populations. Second, we only analyzed the risk of lung cancer, yet prognostic parameters such as overall survival and progression-free survival also warrant additional study. Finally, functional experiments are required to elucidate the underlying disease mechanisms.

Taken together, our results indicate that ERCC5 SNPs have a significant association with lung cancer risk progression. ERCC5 rs4771436 and rs1047768 were found to increase lung cancer risk, especially in men or those aged $\leq 60$ years. These correlations appear to be explained by the distribution of individual SNPs in patients with different clinicopathological parameters. It is to be expected that data from a larger population sample will support these findings, which could then be used to guide the clinical treatment of lung cancer.

\section{Acknowledgements}

This work was supported by grants from the Doctoral Science and Technology Research Startup Fund Project of Liaoning Province of China (2019-BS275), the Science and Technology Fund Project of Liaoning Province of China (20180550318), and Key Laborotary of Tumor Radiosensitization and Normal Tissue Radioprotection of Liaoning Province (2018225102).

\section{Competing Interests}

The authors have declared that no competing interest exists.

\section{References}

1. Xue Q, Liu Z, Feng Z, Xu Y, Zuo W, Wang Q, Gao T, Zeng J, Hu X, Jia F et al: Penfluridol: An antipsychotic agent suppresses lung cancer cell growth and metastasis by inducing G0/G1 arrest and apoptosis. Biomedicine \& pharmacotherapy $=$ Biomedecine \& pharmacotherapie 2020, 121:109598.

2. Lorenzo-González $M$, Ruano-Ravina A, Torres-Durán $M$, Kelsey KT, Provencio M, Parente-Lamelas I, Leiro-Fernández V, Vidal-García I, Castro-Añón O, Martínez C et al: Residential radon, genetic polymorphisms in DNA damage and repair-related. Lung cancer (Amsterdam, Netherlands) 2019, 135:10-15. 
3. Wang L, Wang LL, Shang D, Yin SJ, Sun LL, Wang XY, Ji HB: Gene polymorphism of DNA repair gene $\mathrm{X}$-ray repair cross complementing group 1 and xeroderma pigmentosum group $\mathrm{D}$ and environment interaction in non-small-cell lung cancer for Chinese nonsmoking female patients. The Kaohsiung journal of medical sciences 2019, 35(1):39-48.

4. Yu T, Xue P, Cui S, Zhang L, Zhang G, Xiao M, Zheng X, Zhang Q, Cai Y, Jin C et al: Rs3212986 polymorphism, a possible biomarker to predict smoking-related lung cancer, alters DNA repair capacity via regulating ERCC1 expression. Cancer medicine 2018, 7(12):6317-6330.

5. Anoushirvani AA, Aghabozorgi R, Ahmadi A, Arjomandzadegan M, Khalili S, Sahraei M, Fereydouni T, Khademi Z: The Relationship Between rs3212986C $>$ A Polymorphism and Tumor Stage in Lung Cancer Patients. Cureus 2019, 11(4):e4423.

6. Zhou RM, Li Y, Wang N, Zhang XI, Dong XI, Guo W: Correlation of XPC Ala499Val and Lys939Gln polymorphisms to risks of esophageal squamous cell carcinoma and gastric cardiac adenocarcinoma. Ai zheng $=$ Aizheng $=$ Chinese journal of cancer 2006, 25(9):1113-1119.

7. Jin MH, Oh DY: ATM in DNA repair in cancer. Pharmacology \& therapeutics 2019, 203:107391.

8. Kay J, Thadhani E, Samson L, Engelward B: Inflammation-induced DNA damage, mutations and cancer. DNA repair 2019, 83:102673.

9. Cavic M, Spasic J, Krivokuca A, Boljevic I, Kuburovic M, Radosavljevic D, Jankovic R: TP53 and DNA-repair gene polymorphisms genotyping as a low-cost lung adenocarcinoma screening tool. Journal of clinical pathology 2019, 72(1):75-80.

10. Chaszczewska-Markowska M, Kosacka M, Chryplewicz A, Dyła T, Brzecka A, Bogunia-Kubik K: ECCR1 and NFKB2 Polymorphisms as Potential Biomarkers of Non-small Cell Lung Cancer in a Polish Population. Anticancer research 2019, 39(6):3269-3272.

11. Li W, Zhang M, Huang C, Meng J, Yin X, Sun G: Genetic variants of DNA repair pathway genes on lung cancer risk. Pathology, research and practice 2019, 215(10):152548

12. Martínez-Ramírez OC, Pérez-Morales R, Castro-Hernández C, Gonsebatt ME, Casas-Ávila L, Valdés-Flores M, Petrosyan P, de León-Suárez VP, Rubio J: Association of the Promoter Methylation and the rs12917 Polymorphism of MGMT with Formation of DNA Bulky Adducts and the Risk of Lung Cancer in Mexican Mestizo Population. DNA and cell biology 2019, 38(4):307-313.

13. Zhang $\mathrm{H}$, Li Y, Guo S, Wang $Y$, Wang H, Lu D, Wang J, Jin L, Jiang G, Wu J et al: Effect of ERCC2 rs13181 and rs1799793 polymorphisms and environmental factors on the prognosis of patients with lung cancer. American journal of translational research 2020, 12(10):6941-6953.

14. Bever KM, Le DT: DNA repair defects and implications for immunotherapy. The Journal of clinical investigation 2018, 128(10):4236-4242.

15. Sang L, Lv Z, Sun LP, Xu Q, Yuan Y: Impact of SNP-SNP interactions of DNA repair gene ERCC5 and metabolic gene GSTP1 on gastric cancer/atrophic gastritis risk in a Chinese population. World journal of gastroenterology 2018, 24(5):602-612.

16. Liu Y, Hu Y, Zhang M, Jiang R, Liang C: Polymorphisms in ERCC2 and ERCC5 and Risk of Prostate Cancer: A Meta-Analysis and Systematic Review. Journal of Cancer 2018, 9(16):2786-2794.

17. Kiyohara C, Yoshimasu K: Genetic polymorphisms in the nucleotide excision repair pathway and lung cancer risk: a meta-analysis. International journal of medical sciences 2007, 4(2):59-71.

18. Zhou RM, Niu CX, Wang N, Liu L, Huang X, Chen ZF, Huo XR, Hao YL, Li Y: XPG Gene Polymorphisms and the Risk of Gastric Cardia Adenocarcinoma. Genetic testing and molecular biomarkers 2016, 20(8):432-437.

19. Liu ZQ, Chen GG, Sun RL, Chen C, Lu MY, Guan LF, Chi XL, Jian YQ, Zhu X, Liu RQ et al: XPG rs873601 G>A contributes to uterine leiomyoma susceptibility in a Southern Chinese population. Bioscience reports 2018, 38(5):1-6.

20. Qi L, Yu HQ, Zhang Y, Ding LJ, Zhao DH, Lv P, Wang WY, Xu Y: A Comprehensive Meta-analysis of Genetic Associations Between Key Polymorphic Loci in DNA Repair Genes and Glioma Risk. Molecular neurobiology 2017, 54(2):1314-1325.

21. Jung SW, Park NH, Shin JW, Park BR, Kim CJ, Lee JE, Shin ES, Kim JA, Chung YH: Polymorphisms of DNA repair genes in Korean hepatocellular carcinoma patients with chronic hepatitis B: possible implications on survival. Journal of hepatology 2012, 57(3):621-627.

22. Bai Y, Xu L, Yang X, Hu Z, Yuan J, Wang F, Shao M, Yuan W, Qian J, Ma H et al: Sequence variations in DNA repair gene XPC is associated with lung cancer risk in a Chinese population: a case-control study. BMC cancer 2007, 7:81.

23. Kutob L, Schneider F: Lung Cancer Staging. Surgical pathology clinics 2020, 13(1):57-71.

24. Duran G, Aguin S, Cruz R, Barros F, Giraldez JM, Bernardez B, Lopez-Lopez R, Carracedo A, Lamas MJ: Association of GSTP1 and ERCC1 polymorphisms with toxicity in locally advanced head and neck cancer platinum-based chemoradiotherapy treatment. Head \& neck 2019, 41(8):2704-2715.

25. Borchiellini D, Etienne-Grimaldi MC, Bensadoun RJ, Benezery K, Dassonville O, Poissonnet G, Llorca L, Ebran N, Formento P, Chateau Y et al: Candidate apoptotic and DNA repair gene approach confirms involvement of ERCC1, ERCC5, TP53 and MDM2 in radiation-induced toxicity in head and neck cancer. Oral oncology 2017, 67:70-76.

26. Hui EP, Ma BB, Chan KC, Chan CM, Wong CS, To KF, Chan AW, Tung SY, Ng WT, Cheng AC et al: Clinical utility of plasma Epstein-Barr virus DNA and
ERCC1 single nucleotide polymorphism in nasopharyngeal carcinoma. Cancer 2015, 121(16):2720-2729.

27. Michiels S, Danoy P, Dessen P, Bera A, Boulet T, Bouchardy C, Lathrop M, Sarasin A, Benhamou S: Polymorphism discovery in 62 DNA repair genes and haplotype associations with risks for lung and head and neck cancers. Carcinogenesis 2007, 28(8):1731-1739.

28. Xu Q, Yuan Y, Sun LP, Gong YH, Xu Y, Yu XW, Dong NN, Lin GD, Smith PN, Li RW: Risk of gastric cancer is associated with the MUC1 568 A/G polymorphism. International journal of oncology 2009, 35(6):1313-1320.

29. Motegi A, Masutani M, Yoshioka KI, Bessho T: Aberrations in DNA repair pathways in cancer and therapeutic significances. Seminars in cancer biology 2019, 58:29-46.

30. Zhang X, Crawford EL, Blomquist TM, Khuder SA, Yeo J, Levin AM, Willey JC: Haplotype and diplotype analyses of variation in ERCC5 transcription cis-regulation in normal bronchial epithelial cells. Physiological genomics 2016, 48(7):537-543.

31. Huang Y, Meng C, Long W, Liu Y, Liu Y, Yang J, Yan Z, Yu D, Xiao S: [XP gene polymorphisms and haplotypes with genetic susceptibility to lung cancer]. Wei sheng yan jiu = Journal of hygiene research 2019, 48(6):919-924.

32. Zhang $C$, Liao $Z, Y u$ G, Huang $W$, Song $X$ : Study on association between ERCC5 single nucleotide polymorphism and susceptibility to esophageal cancer. Journal of BUON: official journal of the Balkan Union of Oncology 2017, 22(4):979-984.

33. Hussain SK, Mu LN, Cai L, Chang SC, Park SL, Oh SS, Wang Y, Goldstein BY, Ding BG, Jiang $Q$ et al: Genetic variation in immune regulation and DNA repair pathways and stomach cancer in China. Cancer epidemiology, biomarkers \& prevention: a publication of the American Association for Cancer Research, cosponsored by the American Society of Preventive Oncology 2009, 18(8):2304-2309.

34. Minina VI, Bakanova ML, Soboleva OA, Ryzhkova AV, Titov RA, Savchenko YA, Sinitsky MY, Voronina EN, Titov VA, Glushkov AN: Polymorphisms in DNA repair genes in lung cancer patients living in a coal-mining region. European journal of cancer prevention: the official journal of the European Cancer Prevention Organisation (ECP) 2019, 28(6):522-528.

35. Shao L, Zuo X, Yang Y, Zhang Y, Yang N, Shen B, Wang J, Wang X, Li R, Jin G et al: The inherited variations of a p53-responsive enhancer in 13q12.12 confer lung cancer risk by attenuating TNFRSF19 expression. Genome biology 2019, 20(1):103. 